\title{
Shot noise in ferromagnet-normal metal systems
}

\author{
Yaroslav Tserkovnyak and Arne Brataas \\ Harvard University, Lyman Laboratory of Physics, Cambridge, Massachusetts 02138
}

\begin{abstract}
A semiclassical theory of the low frequency shot noise in ferromagnet-normal metal systems is formulated. Noncollinear magnetization directions of the ferromagnetic leads, arbitrary junctions and the elastic and inelastic scattering regimes are considered. The shot noise is governed by a set of mesoscopic parameters that are expressed in terms of the microscopic details of the junctions in the circuit. Explicit results in the case of ballistic, tunnel, and diffusive junctions are evaluated. The shot noise, the current and the Fano factor are calculated for a double barrier ferromagnet-normal metal-ferromagnet system. It is demonstrated that the shot noise can have a nonmonotonic behavior as a function of the relative angle between the magnetizations of the ferromagnetic reservoirs.
\end{abstract}

\section{INTRODUCTION}

Magnetoelectronic circuits have recently attracted considerable interest due to their potential for magnetic random access memories, sensors, and for fundamental studies of spin transport in magnetic and nonmagnetic

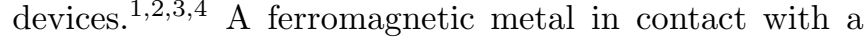
paramagnet can inject spins int the paramagnet or detect spins from the paramagnet. Spin accumulation and its effect on the current-voltage characteristics have been studied thoroughly in double barrier ferromagnet-normal metal-ferromagnet $(F-N-F)$ systems 6, 6

An important parameter in view of applications of spin injection is the noise to signal ratio. The noise furthermore provides additional information on the electronic structure and the nonequilibrium transport processes and that is our main motivation for this study. The calculation and experimental detection of shot noise has been an actize subfield of mesoscopic physics during the last decade. 8 Shot noise in systems consisting of normal metals, superconductors, semiconductors and combinations thereof combined by tunnel barriers, diffusive barriers and ballistic barriers have been studied 8 There have been much less attention on the shot noise in hybrid systems involving ferromagnets.

Only a few studies have been carried out on the effects of the fluctuations in the spin accumulation on the shot noise in ferromagnet - normal metal systems. Bulka et al. computed in Ref. 9 the shot noise in $F-N-F$ double barrier systems in the Coulomb blockade regime when the magnetizations in the ferromagnetic reservoirs are collinear and found pronounced and interesting effects due to the interplay of the spin and charge fluctuations . Their results in the regime when the source-drain bias is larger than the Coulomb charging energy can be understood in terms of well-known results for normal-metal systems for two spin directions. Nowak et al 10 measured electrical noise in ferromagnet-insulator-ferromagnet $(F-I-F)$ systems in the collinear configurations. They also obtained results consistent with a generalization of the results for the normal metal systems. Spin-dependent tunneling through $F-I-F$ systems in the case of noncollinear magnetization configurations were experimentally studied by Moodera et al.11 with results in good agreement with the predictions for the angilar dependence of magnetoresistance by Slonczewski, 12 but no noise measurements were performed. It is our purpose to provide a more general discussion of the shot noise in ferromagnet-normal metal systems where Coulomb charging effects are negligible.

The noise power $S(\omega)$ is defined as

$$
S(\omega)=2 \int_{-\infty}^{\infty} d t e^{-i \omega t}\langle\Delta I(t) \Delta I(0)\rangle,
$$

where $\Delta I(t)=I(t)-\langle I(t)\rangle$ denotes the fluctuation of the current $I(t)$ from its average $\langle I(t)\rangle$. The noise power has contributions from $1 / f$ noise, thermal noise, and shot noise. In equilibrium, the thermal noise is given by the Johnson-Nyquist formula in terms of the conductance of the system. We will consider the nonequilibrium noise contributions in the limit when the bias voltage is larger than the thermal energy $e V \gg k_{\mathrm{B}} T$, so that the thermal Johnson-Nyquist noise can be disregarded. The $1 / f$ noise dominates at very low frequencies or high bias.

Although our theoretical framework allows the calculation of the shot noise in multiterminal systems, we will focus our attention on double barrier systems. Double barrier systems have a characteristic time scale corresponding to the dwell time $\tau_{d}$ of the electron on the node. We will consider the low-frequency shot-noise contribution to the noise power when $\omega \ll \tau_{d}^{-1}$ but the frequency $\omega$ is higher than the frequency at which the $1 / f$ noise dominates. Thus in this regime the low-frequency shot noise is the dominant term and we will set $\omega=0$ in the following and disregard the $1 / f$ noise.

The Landauer-Büttiker (LB) theory of transport in phase-coherent mesoscopic conductors gives simple expressions for the average current 13

$$
I=\frac{e^{2}}{h} V \sum_{n} T_{n},
$$

and the low-frequency shot-noise power

$$
S=\frac{2 e^{3}}{h} V \sum_{n} T_{n}\left(1-T_{n}\right)
$$

where $V$ is the applied voltage and $T_{n}$ (transmission probabilities) are the eigenvalues of $t t^{\dagger}$ composed from 
the transmission matrix $t$ of the junction at Fermi energy. It is assumed that the scattering matrix is energy independent in the energy interval determined by the chemical potentials of the voltage probes. This is easily realized in metallic systems and/or at low bias. Of special interest is the Fano factor which is defined as the ratio between the shot-noise power and the average current: $F=S /(2 e I)$. When the transmission of the particles of charge $e$ is Poissonian (i.e., random) the shot noise is $S=2 e I$, and the Fano factor is $F=1$.

We consider a double barrier $F-N-F$ system as shown in Fig. 1. The system is composed of a chaotic cavity connected to two voltage probes at different chemical potentials through junctions. The left and the right probes are ferromagnetic with magnetization directions $\mathbf{m}_{1}$ and $\mathbf{m}_{2}$, respectively. Our theory is applicable if the following assumptions hold: The spin-flip relaxation time on the normal metal island is much longer than the typical dwell time on the island. The size of each of the two junctions is much smaller than the corresponding phasecoherence length, but the motion of the electrons inside the normal metal island can be elastic or inelastic. The electrons trapped in the cavity dwell long enough so that the scattering in the cavity leads to a homogeneous and isotropic phase-space distribution of electrons inside the cavity. This can be achieved, for example, by scattering off the bulk disorder (diffusive cavity) or by scattering off the irregularities at the surface (ballistic cavity). The theory is applicable if the cavity has negligible resistance compared to the resistances of the junctions, e.g. the voltage only drops across the junctions. Two scattering regimes are considered: quasielastic when $\tau_{d} \ll \tau_{\text {in }}$, and inelastic when $\tau_{\text {in }} \ll \tau_{d}$, where $\tau_{\text {in }}$ is the inelastic scattering time, and $\tau_{d}$ is the dwell time in the cavity.

We will study the influence of the relative difference in the magnetization directions on the shot noise. In the case of collinear magnetizations, the results for the all-normal metal systems can be easily generalized by taking the two spin directions separately into account. The regime of noncollinear magnetization configuration is more interesting and we will present novel nontrivial results for this case. There are, to the best of our knowledge, no systematic predictions for the Fano factor in this regime. We will also present results for the shot noise in other transport regimes than previously studied for $F-N$ systems. In addition to the tunneling regime, already discussed in some limits, 10 we will extend the analysis to ballistic and diffusive junctions. Different regimes of scattering on the normal metal node will also be studied: The elastic scattering regime and the inelastic scattering regime.

Let us first review the results for transpgrt through a single barrier in all-normal metal systems. The Fano factor in the cases of ballistic $(B)$, diffusive $(D)$, and tunnel $(T)$ junctions are

$$
\begin{aligned}
& F_{B}=0, \\
& F_{D}=1 / 3,
\end{aligned}
$$

$$
F_{T}=1 .
$$

In the ballistic and tunnel regimes the result follows directly from the LB theory (2,3), since $T_{n} \ll 1$ for tunnel junctions and $T_{n}=0$ or $T_{n}=1$ for ballistic junctions. The $1 / 3$ noise suppression in diffusive junctions is due to the bimodal form of the distribution function of transmission eigenvalues.

Next, let us review the results for all-normal metal double barrier systems in the elastic transport regime. The Fano factor is $F=\left(R_{1}^{3} F_{1}+R_{2}^{3} F_{2}+R_{1}^{2} R_{2}+R_{1} R_{2}^{2}\right) /\left(R_{1}+\right.$ $\left.R_{2}\right)^{3}$, where $F_{i}\left(R_{i}\right)$ is the Fano factor (resistance) of the $i$ th junction. 14 For ballistic, diffusive and tunnel junctions the Fano factors for double barrier systems are

$$
\begin{aligned}
& F_{B, 2}^{\mathrm{el}}=\frac{R_{1} R_{2}}{\left(R_{1}+R_{2}\right)^{2}}, \\
& F_{D, 2}^{\mathrm{el}}=1 / 3, \\
& F_{T, 2}^{\mathrm{el}}=\frac{R_{1}^{2}+R_{2}^{2}}{\left(R_{1}+R_{2}\right)^{2}} .
\end{aligned}
$$

In the ballistic case (77), each junction exhibits zero shot noise, but the shot noise of the double barrier system is finite since the chaotic nodes have a partial (fluctuating) occupation of the energy levels in the nonequilibrium situation even at zero temperature. Consequently, although the direct contribution to the shot noise from a single junction vanishes, the effective 'thermal' contribution to the noise is finite. The elastic chaotic cavity was recently realized as a quantum dot connected to voltage probes with two point contacts 15 and the Fano factor (7) was experimentally confirmed. For diffusive junctions, the Fano factor (8) is the same as for a single junction (5) because an elastic double diffusive barrier system can be seen as a single diffusive junction. For tunnel junctions, the Fano factor (9) attains its minimum, $F_{T, 2}^{\mathrm{el}}=1 / 2$, when the barriers are symmetric. For completely asymmetric barriers, the system is governed by the barrier with the lowest transmission only and therefore the Fano factor is $F_{T, 2}^{\mathrm{el}}=1$ as in the case of a single tunnel barrier.

In the inelastic transport regime the Fano factor for a double barrier system is $145=\left(R_{1}^{2} F_{1}+R_{2}^{2} F_{2}\right) /\left(R_{1}+\right.$ $\left.R_{2}\right)^{2}$. Because the energy level occupation numbers in the node obey the Fermi-Dirac (FD) distribution with a fluctuating chemical potential, the Fano factor of the entire system is a linear combination of the Fano factors of the two junctions. Ballistic, diffusive, and tunnel junctions then yield, respectively,

$$
\begin{aligned}
& F_{B, 2}^{\mathrm{in}}=0 \\
& F_{D, 2}^{\mathrm{in}}=\frac{1}{3} \frac{R_{1}^{2}+R_{2}^{2}}{\left(R_{1}+R_{2}\right)^{2}}, \\
& F_{T, 2}^{\mathrm{in}}=\frac{R_{1}^{2}+R_{2}^{2}}{\left(R_{1}+R_{2}\right)^{2}} .
\end{aligned}
$$

In the case of tunnel junctions, we get the same result as in the elastic regime. The Fano factor in the diffusive 
double barrier system differs from $1 / 3$, which is a universal noise suppression factor in elastic diffusive wires. $1 / 3$ suppression is restored only for a completely asymmetric system governed by the lowest transmission barrier. Finally, ballistic system has vanishing noise because the node has a FD distribution of electrons.

We will generalize the results above to ferromagnetnormal metal systems with noncollinear magnetization directions. The manuscript is organized in the following way. In Sec. II we describe the circuit theory used to analyze multiterminal $F-N$ systems and relate the current and its fluctuations through a junction to the adjacent distribution functions and the scattering matrices for spin-up and spin-down electrons. We compute the Fano factor for double barrier junction systems in Sec. [II]. Our conclusions can be found in Sec. [V].

\section{CIRCUIT THEORY}

Nazarov realized that transport in hybrid superconductor-normal metal systems can be understood in terms of a generalized circuit theory similar to Ohm's law 16 In a similar way a circuit theory for ferromagnet-normal metal systems was formulated by Brataas, Nazarov, and Bauer.6 We extend the circuit theory of transport through ferromagnetic-normal metal systems to the calculation of shot noise. A (magneto)electronic circuit is divided into junctions (resistive elements), nodes (low impedance interconnectors), and reservoirs (voltage probes at thermal equilibrium). The theory is applicable when the junctions limit the electric current and the nodes are characterized by a distribution function which is constant in position space (this assumption can be relaxed, see Ref. 17) and isotropic in momentum space. It is assumed that there are no spin-flip processes in the nodes. In the following discussion the nodes are taken to be normal metal chaotic cavities. Each voltage probe is ferromagnetic, connected to the nodes through leads carrying current and spin current. The current in the circuit depends on the relative magnetization orientations in the probes 6

The current in the circuit can be derived by using the Keldysh Green function technique. 6 We obtain the same result using LB approach generalized to describe circuits with one or more terminals (the nodes) with nonequilibrium spin distribution functions. The assumption of the LB theory is that particles exiting a probe have the same occupation of the energy levels as the particles inside the probe and the occupation numbers of the incoming particles are uniquely determined by the probes that supply them and the scattering properties of the junctions. We generalize the LB expression for the current operator from a normal metal node into a junction to a $2 \times 2$ current operator in spin space in order to also describe spin currents collinear and noncollinear to the magnetization directions in the adjacent ferromagnets. The $2 \times 2$ current operator for the particle flow from node 2 to node 1 is

$$
\begin{aligned}
\hat{I}^{\alpha \beta}(t)= & \frac{e}{h} \sum_{n} \int d E d E^{\prime} e^{i\left(E-E^{\prime}\right) t / \hbar} \\
& {\left[a_{\beta n, 1}^{\dagger}(E) a_{\alpha n, 1}\left(E^{\prime}\right)-b_{\beta n, 1}^{\dagger}(E) b_{\alpha n, 1}\left(E^{\prime}\right)\right], }
\end{aligned}
$$

where $a_{\alpha n, i}^{\dagger}(E)$ creates a spin- $\alpha$ electron with energy $E$ leaving the $i$ th node through the $n$th transverse mode and $b_{\alpha n, i}^{\dagger}(E)$ creates a spin- $\alpha$ electron with energy $E$ entering the $i$ th node through the $n$th transverse mode. The current operator is $I=\sum_{\alpha} \hat{I}^{\alpha \alpha}$ and the spin-current operator is $\hat{\mathbf{I}}_{s}=-\hbar /(2 e) \sum_{\alpha \beta} \hat{\boldsymbol{\sigma}}_{\alpha \beta} \hat{I}^{\beta \alpha}$, where $\hat{\boldsymbol{\sigma}}=\left(\hat{\sigma}_{x}, \hat{\sigma}_{y}, \hat{\sigma}_{z}\right)$ is a vector of the Pauli matrices. If the scattering matrices of the junctions are known then we can express annihilation operators $b_{\alpha n, i}(E)$ in terms of the operators $a_{\alpha n, i}(E)$. Expectation values involving operators $a_{\alpha n, i}^{\dagger}(E)$ and $a_{\alpha n, i}(E)$ can then be evaluated in terms of the distribution functions in the adjacent nodes and we can calculate both the current and the shot noise 13

Let us consider a node that is connected to several other nodes via junctions. In the low frequency, longtime limit $\omega \ll 1 / \tau_{d}$ the charge and spin in the node are conserved at every instance of time

$$
\sum_{i} \hat{I}_{i}(t)=0
$$

where $\hat{I}_{i}$ is the current flowing out of the node through the $i$ th junction at time $t$ and the index $i$ runs over all junctions connected to the node. Due to the charge and spin conservation (14), the distribution function on the normal metal node has two contributions: A stationary part $\hat{f}_{N}(E)$ and a small fluctuating part $\delta \hat{f}_{N}(E, t)$ caused by the discreteness of the charges and spins that enter and leave the island. Using the isotropy assumption in momentum space, the stationary part $\hat{f}_{N}(E)$ is defined by $\left\langle a_{\alpha m}^{\dagger}(E) a_{\beta n}\left(E^{\prime}\right)\right\rangle=\delta_{n m} \delta\left(E-E^{\prime}\right) \hat{f}_{N}^{\beta \alpha}(E)$, where $a_{\alpha m}^{\dagger}$ creates a spin- $\alpha$ particle in the $m$ th quantum state of the node. The distribution function $\hat{f}_{N}(E)$ is a $2 \times 2$ Hermitian matrix in spin space to allow a nonequilibrium spin accumulation on the normal metalnode.

Following Beenakker and Büttiker, 14 we separate the time-dependent current fluctuations $\Delta \hat{I}_{i}(t)$ in the $i$ th junction into two contributions. The first contribution $\delta \hat{I}_{i}(t)$ is due to the intrinsic noise in the junction if the fluctuating term $\delta \hat{f}_{N}(E, t)$ of the distribution function on the node is neglected. This is the only relevant term for the shot noise for a single junction connected to reservoirs that have stationary distribution functions. The second contribution is due to the small fluctuation in the occupation of the energy levels on the node $\delta \hat{f}_{N}(E, t)$.

Spin and charge conservation (14) dictate the average occupation of the energy levels in the node in terms of the average distribution functions in the adjacent nodes. 
From Eq. (14) the total fluctuations of the current vanish

$$
\sum_{i} \Delta \hat{I}_{i}(t)=0
$$

Consequently, small fluctuations of the occupation of the energy levels are needed to compensatefor the intrinsic current fluctuations in the junctions. 14 The total fluctuation $\Delta \hat{I}_{i}(t)$ is a sum of these two contributions:

$$
\Delta \hat{I}_{i}(t)=\delta \hat{I}_{i}(t)+\sum_{j} \frac{\delta\left\langle\hat{I}_{i}\right\rangle}{\delta\left\langle\hat{f}_{j}\right\rangle} \delta \hat{f}_{j}(t),
$$

where $j$ runs over the nodes adjacent to the $i$ th junction. The calculation of the term proportional to $\delta \hat{f}_{j}(t)$ resembles the case of all-normal metal systems, but is now generalized to describe the $2 \times 2$ spin-space matrices. The calculation of this term is straightforward, but tedious since it requires solving for the $2 \times 2$ distribution functions in the circuit. The explicit result for the total fluctuation in the current $\Delta \hat{I}_{i}(t)$ in terms of the intrinsic fluctuation in the current through the separate junctions $\delta \hat{I}_{i}(t)$ is shown below in Eq. (30) for symmetric double barrier systems.

The ferromagnetic reservoirs are in thermal equilibrium characterized by equilibrium FD distribution functions independent of time. In contrast, the normal metal islands are isolated from the thermal baths and have (fluctuating) nonequilibrium distribution functions required for current conservation. The normal metal nodes can be viewed as fictitious probes.14 They are characterized by an isotropic and homogeneous distribution function like the true probes (i.e., probes in local thermal equilibrium), but the (fluctuating) distribution function does not have to be of the FD form.

\section{A. Average current}

Naturally, we obtain here the same result for the average current by using the LB formalism as from the Keldysh formalism 6 since they are identical when there is no inelastic scattering in the junctions. For completeness we show the result for the average current (per unit of energy, at a given energy) between a ferromagnetic node and a normal metal node at the normal metal side 9

$$
\begin{gathered}
\hat{\imath}=\frac{e}{h}\left[g^{\uparrow} \hat{u}^{\uparrow}\left(\hat{f}_{F}-\hat{f}_{N}\right) \hat{u}^{\uparrow}+g^{\downarrow} \hat{u}^{\downarrow}\left(\hat{f}_{F}-\hat{f}_{N}\right) \hat{u}^{\downarrow}\right. \\
\left.-g^{\uparrow \downarrow} \hat{u}^{\uparrow} \hat{f}_{N} \hat{u}^{\downarrow}-g^{\downarrow \uparrow} \hat{u}^{\downarrow} \hat{f}_{N} \hat{u}^{\uparrow}\right],
\end{gathered}
$$

where $\hat{u}^{\uparrow}=(\hat{1}+\hat{\boldsymbol{\sigma}} \cdot \mathbf{m}) / 2$ and $\hat{u}^{\downarrow}=(\hat{1}-\hat{\boldsymbol{\sigma}} \cdot \mathbf{m}) / 2$ are projection matrices, $\hat{f}_{N}$ is the average distribution function in the normal metal node, $\hat{f}_{F}$ is the equilibrium distribution function in the ferromagnetic probe, and the conductances are defined in terms of the reflection matrices for electrons incoming from the normal metal node

$$
\hat{g}=\left(\begin{array}{cc}
g^{\uparrow} & g^{\uparrow \downarrow} \\
g^{\downarrow \uparrow} & g^{\downarrow}
\end{array}\right)
$$

$$
=\sum_{n m}\left(\begin{array}{cc}
\delta_{n m}-\left|r_{n m}^{\uparrow}\right|^{2} & \delta_{n m}-r_{n m}^{\uparrow}\left(r_{n m}^{\downarrow}\right)^{*} \\
\delta_{n m}-r_{n m}^{\downarrow}\left(r_{n m}^{\uparrow}\right)^{*} & \delta_{n m}-\left|r_{n m}^{\downarrow}\right|^{2}
\end{array}\right) .
$$

For energies much less than the Fermi energy, the conductance (18) is energy independent. Therefore, the elastic and inelastic transport regimes are equivalent for the average current. However, the shot noise differs in these transport regimes, sipce it is sensitive to the the energyresolved distribution.

\section{B. Shot noise}

The starting point in calculating the noise is the relation between the fluctuation of the current in a junction and of the distribution function in the fictitious probes adjacent to the junction (16). The first term on the right-hand side of the Eq. (16) is a spontaneous Langevin source. The second term (16) can be found in terms of the Langevin source by using Eq. (15) for the instantaneous conservation of spin and Eq. (17) for the current. The problem of calculating the noise is then reduced to finding the correlator of the Langevin sources $\delta \hat{I}_{i}$ in terms of the scattering matrices of the junctions and the distribution functions in the adjacent nodes and reservoirs.

The correlators of the Langevin sources can be found by generalizing the LB theory for all-normal metal systems. We expand the current in terms of the creation and annihilation operators, using Eq. (13), and then determine particle and spin fluctuations

$$
\begin{aligned}
& \left\langle a_{\alpha k}^{\dagger}\left(E_{1}\right) a_{\beta l}\left(E_{2}\right) a_{\gamma m}^{\dagger}\left(E_{3}\right) a_{\delta n}\left(E_{4}\right)\right\rangle \\
& \quad-\left\langle a_{\alpha k}^{\dagger}\left(E_{1}\right) a_{\beta l}\left(E_{2}\right)\right\rangle\left\langle a_{\gamma m}^{\dagger}\left(E_{3}\right) a_{\delta n}\left(E_{4}\right)\right\rangle \\
& \quad=\delta_{k n} \delta_{l m} \delta\left(E_{1}-E_{4}\right) \delta\left(E_{2}-E_{3}\right) \\
& \quad \times \hat{f}_{n}^{\delta \alpha}\left(E_{1}\right)\left[1-\hat{f}_{m}^{\beta \gamma}\left(E_{2}\right)\right],
\end{aligned}
$$

where the Greek subscripts denote spin indices, and the Latin subscripts include both the transverse mode index and the probe label. However, the distribution function in the probe $\hat{f}_{n}$ is independent of the transverse mode and $n$ here denotes only the probe label.

A similar analysis used to calculate shot noise in nonmagnetic circuits. 14 Our treatment of spin transport is a generalization to include the spin degree of freedom. In addition to instantaneous conservation of charge in the nodes, we use instantaneous conservation of spin. Both conditions are satisfied when the frequency is lower than the inverse dwell time of a particle in the node, $\omega \tau_{d} \ll 1$.

When the inelastic scattering time is much longer than the transport dwell time, the energy of a quasiparticle is conserved. In this regime both current and noise can be calculated at each energy level and then integrated over the energy to get the final result.

The inelastic regime is achieved when the inelastic scattering time is shorter than the transport dwell time. 
When a particle is trapped in a node, it is assumed to drop to the lowest energy state allowed without a spin flip. As a result, there will be a direction of spin accumulation in the node with FD distribution for electrons polarized parallel and antiparallel to it (with different chemical potentials $\mu^{\uparrow}$ and $\mu^{\downarrow}$, respectively). The direction of spin accumulation and its magnitude are determined by the transport rates integrated over all energies.

\section{Junction conductance and shot-noise matrices}

Because the current (13) is expressed as a linear combination of products of two creation and annihilation operators, the current in the circuit is completely deter- mined by $2 \times 2$ conductance matrices (18), elements of which can be expressed as traces of two reflection matrices. Shot noise is quadratic in current, and we expect that it will be governed by similar traces with four reflection and transmission matrices. We show that this is indeed true with some simplifications. First, because ferromagnetic probes are in local thermal equilibrium, the new independent parameters can be expressed in terms of the traces of four reflection matrices only. Second, only the traces of the form $\operatorname{Tr}\left[r^{\alpha}\left(r^{\alpha^{\prime}}\right)^{\dagger} r^{\beta}\left(r^{\beta^{\prime}}\right)^{\dagger}\right]$ enter the shot noise, where $\alpha, \alpha^{\prime}, \beta$, and $\beta^{\prime}$ denote the spin. The new set of parameters describing the shot noise of a junction can therefore be grouped into a $4 \times 4$ Hermitian matrix $\hat{s}$ :

$$
\hat{s}=\operatorname{Tr}\left[\left(\begin{array}{cccc}
\hat{1} & \hat{1} & \hat{1} & \hat{1} \\
\hat{1} & \hat{1} & \hat{1} & \hat{1} \\
\hat{1} & \hat{1} & \hat{1} & \hat{1} \\
\hat{1} & \hat{1} & \hat{1} & \hat{1}
\end{array}\right)-\left(\begin{array}{cccc}
r^{\uparrow}\left(r^{\uparrow}\right)^{\dagger} r^{\uparrow}\left(r^{\uparrow}\right)^{\dagger} & r^{\uparrow}\left(r^{\uparrow}\right)^{\dagger} r^{\uparrow}\left(r^{\downarrow}\right)^{\dagger} & r^{\uparrow}\left(r^{\downarrow}\right)^{\dagger} r^{\uparrow}\left(r^{\uparrow}\right)^{\dagger} & r^{\uparrow}\left(r^{\downarrow}\right)^{\dagger} r^{\uparrow}\left(r^{\downarrow}\right)^{\dagger} \\
r^{\uparrow}\left(r^{\uparrow}\right)^{\dagger} r^{\downarrow}\left(r^{\uparrow}\right)^{\dagger} & r^{\uparrow}\left(r^{\uparrow}\right)^{\dagger} r^{\downarrow}\left(r^{\downarrow}\right)^{\dagger} & r^{\uparrow}\left(r^{\downarrow}\right)^{\dagger} r^{\downarrow}\left(r^{\uparrow}\right)^{\dagger} & r^{\uparrow}\left(r^{\downarrow}\right)^{\dagger} r^{\downarrow}\left(r^{\downarrow}\right)^{\dagger} \\
r^{\downarrow}\left(r^{\uparrow}\right)^{\dagger} r^{\uparrow}\left(r^{\uparrow}\right)^{\dagger} & r^{\downarrow}\left(r^{\uparrow}\right)^{\dagger} r^{\uparrow}\left(r^{\downarrow}\right)^{\dagger} & r^{\downarrow}\left(r^{\downarrow}\right)^{\dagger} r^{\uparrow}\left(r^{\uparrow}\right)^{\dagger} & r^{\downarrow}\left(r^{\downarrow}\right)^{\dagger} r^{\uparrow}\left(r^{\downarrow}\right)^{\dagger} \\
r^{\downarrow}\left(r^{\uparrow}\right)^{\dagger} r^{\downarrow}\left(r^{\uparrow}\right)^{\dagger} & r^{\downarrow}\left(r^{\uparrow}\right)^{\dagger} r^{\downarrow}\left(r^{\downarrow}\right)^{\dagger} & r^{\downarrow}\left(r^{\downarrow}\right)^{\dagger} r^{\downarrow}\left(r^{\uparrow}\right)^{\dagger} & r^{\downarrow}\left(r^{\downarrow}\right)^{\dagger} r^{\downarrow}\left(r^{\downarrow}\right)^{\dagger}
\end{array}\right)\right],
$$

where the trace is taken for each element of the $4 \times 4$ matrix (each of which is an $M \times M$ matrix in the basis of the $M$ transverse modes) inside the square brackets, i.e., the result of the operation is a $4 \times 4$ matrix of complex numbers.

Only four off-diagonal elements and three diagonal elements are independent in the shot-noise matrix $\hat{s}$. Therefore, in general, we introduce 7 new parameters: The real parameters $s_{\uparrow}, s_{\uparrow \downarrow}, s_{\downarrow}$, and the complex quantities $s_{+}$, $s_{-}, s_{0}, \bar{s}_{0}$,

$$
\hat{s}=\left(\begin{array}{cccc}
s_{\uparrow} & s_{+} & s_{+} & \bar{s}_{0} \\
s_{+}^{*} & s_{\uparrow \downarrow} & s_{0} & s_{-} \\
s_{+}^{*} & s_{0}^{*} & s_{\uparrow \downarrow} & s_{-} \\
\bar{s}_{0}^{*} & s_{-}^{*} & s_{-}^{*} & s_{\downarrow}
\end{array}\right) .
$$

These parameters (21) and the conductance parameters (18) completely determine the current and noise in the system. Scaling of all $\hat{r}$ and $\hat{s}$ matrices of a given system by the same factor does not change the Fano factor.

We proceed with the explicit evaluation of the conductance $\hat{g}$ and shot-noise $\hat{s}$ matrices in the cases of tunnel, diffusive and ballistic junctions below and then apply our theory to two-terminal double barrier ferromagneticnormal-ferromagnetic $(F-N-F)$ systems. The results for the conductance matrix $\hat{g}$ have been derived in Ref. 6 and we briefly reiterate these results here for completeness. The shot-noise matrix $\hat{s}$ has not been studied before.

\section{Ballistic junctions}

Because the transverse momentum is conserved in ballistic junctions, the reflection matrices $\left(r^{\uparrow}\right.$ and $\left.r^{\downarrow}\right)$ are diagonal in the hasis of the transverse modes. Simplifying the situation, 18 we assume that the diagonal components of the reflection matrices can attain only two values: Full transmission 0 or no transmission 1. From Eqs. (2), (3) we see that the Fano factor for a single ballistic junction vanishes. It is not the case in a double barrier system, as discussed in the Introduction.

The conductance $\hat{g}$ and shot-noise $\hat{s}$ matrices for a single junction are in this case

$$
\begin{aligned}
& \hat{g} / g=\left(\begin{array}{cc}
1+p & 1+|p| \\
1+|p| & 1-p
\end{array}\right),
\end{aligned}
$$

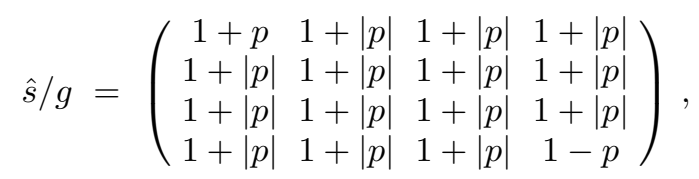

where $g=\left(g^{\uparrow}+g^{\downarrow}\right) / 2$ is the average conductance and $p=\left(g^{\uparrow}-g^{\downarrow}\right) /\left(g^{\uparrow}+g^{\downarrow}\right)$ is the relative polarization. If all the junctions are the same, noise suppression will depend only on the relative polarization $p$ and the relative magnetization orientations.

\section{Diffusive junctions}

The conductance for diffusive junctions can be found with different methods. One possibility is to solve the 
diffusion equation with the proper boundary conditions as was done in Ref. 6 to find the mixing conductance $g^{\uparrow \downarrow}$. An alternative approach is to evaluate the conductance matrix by using random matrix theory (RMT) in the semiclassical approximation.19.20 We will use the latter approach here, because it considerably simplifies the calculation of the shot-noise parameters.

We assume that the junction consists of two connected parts as shown in Fig. 2. The first, normal metal part is described by a single scattering matrix for both spin$\uparrow$ and spin- $\downarrow$ electrons. The second, ferromagnetic part is described by two independent scattering matrices, one for spin- $\uparrow$ and one for spin- $\downarrow$ electrons. It is assumed that there are no correlations between the scattering matrices of the spin- $\uparrow$ and spin- $\downarrow$ electrons in the ferromagnetic part. However the up- and down-spin parts of the total scattering matrix of the combined normal metal and ferromagnetic system are correlated since both spin directions see the same scattering centers in the normal metal part. Scattering at the $F-N$ boundary is disregarded since it is assumed that the total resistance is dominated by the diffuse normal metal and ferromagnetic metal parts of the junction. The total reflection matrix $r^{\alpha}$ for spin- $\alpha$ electrons can then be found by concatenating the normal metal and ferromagnetic parts

$$
r^{\alpha}=r_{N}+t_{N}^{\prime} r_{F}^{\alpha} \sum_{n=0}^{\infty}\left(r_{N}^{\prime} r_{F}^{\alpha}\right)^{n} t_{N} .
$$

We apply the standard polar decomposition for reflection matrices of the spin- $\alpha$ electrons inside the ferromagnetic part:21 $r_{F}^{\alpha}=i U^{\alpha} \sqrt{T_{F}^{\alpha}} U^{\prime \alpha}$, where $U^{\alpha}, U^{\prime \alpha}$ are $M \times M$ mitary matrices, which in the isotropic approximation 21 are uniformly distributed in the group $U(M)$. In the case of time-reversal symmetry, these matrices are related by transposition, $U^{\prime \alpha}=U^{\alpha^{\mathrm{T}}} . T_{F}^{\alpha}$ is a diagonal matrix containing the eigenvalues of $t_{F}^{\alpha}\left(t_{F}^{\alpha}\right)^{\dagger}$. After inserting these reflection matrices into the expansion (22), we perform the averaging of traces entering conductance $\hat{g}$ and shot-noise $\hat{s}$ matrices over the scattering matrices in the ferromagnetic part. To this end we use the semiclassical result for traces of matrices from RMT 19

In order to perform the averaging, expressions of the following form must be evaluated.

$$
\rho \equiv \operatorname{Tr}\left[(A \alpha B \beta \cdots \eta C)\left(F^{\dagger} \omega^{\dagger} \cdots \delta^{\dagger} E^{\dagger} \gamma^{\dagger} D^{\dagger}\right)\right],
$$

where $A, B, C, D, E, F$ are some fixed $M \times M$ matrices and $\alpha, \beta, \eta, \gamma, \delta, \omega$ are unitar-matrices uniformly distributed in $U(M)$. It was shown 19 that if the ordered sets $\alpha, \beta, \ldots, \eta$ and $\gamma, \delta, \ldots, \omega$ are such that any two closest neighbors are independent (or related by transposition, i.e., $\alpha=\beta^{\mathrm{T}}$ ), then, to the leading order in $M$, this trace is nonzero only if $A=D, B=E, \ldots, C=F$ and $\alpha=\gamma$, $\beta=\delta, \ldots, \eta=\omega$. If these conditions are satisfied, the trace (23) equals (to the leading semiclassical order in the inverse number of transverse modes $1 / M$ )

$$
\rho=M \frac{1}{M} \operatorname{Tr}\left[A A^{\dagger}\right] \frac{1}{M} \operatorname{Tr}\left[B B^{\dagger}\right] \cdots \frac{1}{M} \operatorname{Tr}\left[C C^{\dagger}\right] .
$$

This is sufficient to calculate both the conductance $\hat{g}$ and the shot-noise $\hat{s}$ matrices in terms of the conductance of the normal metal part and up- and down-spin conductances in the ferromagnetic part, after we resolve two problems. First, we encounter the task of calculating the trace $\operatorname{Tr}\left[t_{N} t_{N}^{\dagger} t_{N} t_{N}^{\dagger}\right]$. Second, the two $\hat{s}$ matrix elements $\hat{s}_{\uparrow}=\operatorname{Tr}\left[\hat{1}-r^{\uparrow}\left(r^{\uparrow}\right)^{\dagger} r^{\uparrow}\left(r^{\uparrow}\right)^{\dagger}\right]$ and $\hat{s}_{\downarrow}=\operatorname{Tr}\left[\hat{1}-r^{\downarrow}\left(r^{\downarrow}\right)^{\dagger} r^{\downarrow}\left(r^{\downarrow}\right)^{\dagger}\right]$ pose a challenge, because some of the terms in their expansions [following Eq. (22)] contain correlations between random matrices which invalidate the assumptions of the semiclassical result (24). We calculate these three quantities using the Boltzmann-Langevin approach. It was shown in Ref. 22 that in all-normal metal systems the Fano factor has a universal 1/3 suppression in diffusive junctions of arbitrary shape, dimension, and conductivity distribution. This is directly applicable to the case at hand because in evaluating $\operatorname{Tr}\left[t_{N} t_{N}^{\dagger} t_{N} t_{N}^{\dagger}\right]$ and $\operatorname{Tr}\left[r^{\alpha}\left(r^{\alpha}\right)^{\dagger} r^{\alpha}\left(r^{\alpha}\right)^{\dagger}\right]$ we deal with collinear transport. Therefore we find

$$
\begin{aligned}
\operatorname{Tr}\left[t_{N} t_{N}^{\dagger} t_{N} t_{N}^{\dagger}\right] & =\frac{2}{3} g_{N}, \\
\hat{s}_{\uparrow} & =\frac{4}{3} g_{\uparrow}, \\
\hat{s}_{\downarrow} & =\frac{4}{3} g_{\downarrow} .
\end{aligned}
$$

Now we use the expansion (22), the semiclassical result (24), and the results (25), (26), and (27) to find the conductance and shot-noise matrices

$$
\begin{aligned}
\hat{g} / g= & \left(\begin{array}{ccc}
1+p & g_{N} / g \\
g_{N} / g & 1-p
\end{array}\right) \\
\frac{3}{2} \hat{s} / g= & \left(\begin{array}{cccc}
2(1+p) & 1+p & 1+p & 0 \\
1+p & 1 & 1 & 1+p \\
1+p & 1 & 1 & 1+p \\
0 & 1+p & 1+p & 2(1+p)
\end{array}\right) \\
& +g_{N} / g\left(\begin{array}{cccc}
0 & 1 & 1 & 2 \\
1 & 0 & 0 & 1 \\
1 & 0 & 0 & 1 \\
2 & 1 & 1 & 0
\end{array}\right) \\
& -g / g_{N}\left(\begin{array}{cccc}
0 & 0 & 0 & 0 \\
0 & 1-p^{2} & 1-p^{2} & 0 \\
0 & 1-p^{2} & 1-p^{2} & 0 \\
0 & 0 & 0 & 0
\end{array}\right)
\end{aligned}
$$

where $g_{N}$ is the conductance of the normal metal part of the junction, $g=\left(g^{\uparrow}+g^{\downarrow}\right) / 2$, and $p=\left(g^{\uparrow}-g^{\downarrow}\right) /\left(g^{\uparrow}+g^{\downarrow}\right)$. The spin-dependent conductance $g^{\uparrow}=\left(g_{F}^{\uparrow} g_{N}\right) /\left(g_{F}^{\uparrow}+g_{N}\right)$ and $g_{F}^{\downarrow}=\left(g_{F}^{\downarrow} g_{N}\right) /\left(g_{F}^{\downarrow}+g_{N}\right)$ are given by Ohm's law in terms of the spin-dependent conductance of the ferromagnet $\left(g_{F}^{\uparrow}\right.$ and $\left.g_{F}^{\downarrow}\right)$ and the conductance of the normal metal $\left(g_{N}\right)$. Two parameters govern the Fano factor if all junctions are the same, the relative polarization $p$ and the fraction of the conductance of the normal metal part to the average conductance $g_{N} / g$. The result for the conductance matrix (28) using RMT agrees with the calculation using the diffusion equation in Ref. 6 . 


\section{Tunnel junctions}

The transmission coefficients are exponentially small in tunnel junctions. We expand $\hat{r}$ and $\hat{s}$ in terms of the small quantities $\delta r_{n m}^{\alpha}=\delta_{n m}-r_{n m}^{\alpha}$ and keep only the lowest order nonvanishing terms. We also assume that the reflection coefficients have random phases. Firstprinciples band-structure calculations confirm this for realistic systems.23 An important result can be drawn from the randomness of the phases of the reflection coefficients: The imaginary part of the mixing conductance 23 and, similarly, the shot-noise parameters vanishes. Using this, we can express the conductance and shot-noise parameters as

$$
\begin{aligned}
\hat{g} / g & =\left(\begin{array}{cc}
1+p & 1 \\
1 & 1-p
\end{array}\right) \\
\hat{s} / g & =\left(\begin{array}{cccc}
1+2 p & 1+p & 1+p & 1 \\
1+p & 1 & 1 & 1-p \\
1+p & 1 & 1 & 1-p \\
1 & 1-p & 1-p & 1-2 p
\end{array}\right)
\end{aligned}
$$

The parameters only depend on the average conductance $g$ and the relative polarization of the junctions $p$.

\section{SYMMETRIC $F-N-F$ DOUBLE BARRIER}

The theory developed above can be used to analyze the shot noise in complicated many-terminal devices containing many normal metal nodes and ferromagnetic reservoirs. Such an analysis has already been performed for the current through two-terminal double barrier ferromagnet-normal metal-ferromagnet systems, a novel three-terminal ferromagnet-normal metal- ferromagnet "spin-flip" transistor and a generalization of Johnson's three-terminal spin-transistor.6 We have seen that, in general, there are 4 conductance parameters and 11 shot-noise parameters that completely describe each junction in the system. The Fano factor defined as the ratio between the shot noise and the current through a junction thus depends on $15 N-1$ parameters, where $N$ is the number of different junctions. (The Fano factor is invariant under the scaling of all parameters and, therefore, we can set any one of the $15 N$ parameters to be unity and correspondingly scale the remaining $15 N-1$ parameters.) Clearly, we cannot explore all possible systems, but we will here illustrate the usefulness of the semiclassical theory of shot noise to study the most simple system of spin accumulation: A two-terminal double barrier ferromagnet-normal metal-ferromagnet system. We will furthermore assume that the system is symmetric and study the regime of ballistic, diffusive, and tunnel junctions. Besides, we will investigate the elastic and inelastic transport regimes separately. The number of independent parameters (defining the angular dependence of the Fano factor) then reduces to 1 (ballistic), 2 (diffusive), and 1 (tunnel), which considerably simplifies the analysis. The results for the current and the shot noise in the collinear configuration, when the magnetizations of the left and the right probes are parallel or antiparallel, can be easily deduced from a two channel model of the corresponding all-normal metal system. All our results in the elastic and inelastic transport regimes and for ballistic, diffusive, and tunnel junctions agree with these known results. The results when the magnetizations are noncollinear are novel. We demonstrate that the Fano factor can exhibit a nonmonotonic dependence on the relative angle between the magnetizations in the ferromagnets. This can be used experimentally to obtain more information about the spin accumulation and the nature of the junctions including their polarizations.

We assume that both ferromagnetic reservoirs are held at zero temperature but at different chemical potentials $\mu_{0}$ and $\mu_{0}+|e V|$, where $V$ is the applied constant voltage bias. We denote $\hat{f}_{N}^{\mathrm{a}}$ the energy-averaged distribution function in the normal metal island for energies between the chemical potentials of the reservoirs. $\hat{f}_{N}^{\mathrm{a}}$ is a function of the conductance matrix $\hat{g}$, the relative angle between the magnetizations of the ferromagnets, and the applied voltage bias and can be found by the average charge and spin conservation in the island as outlined in Ref. 6 and is the same in both elastic and inelastic regimes.

We use Eq. (17) for the current flowing through the left and right junctions and Eqs. (16), (15) for the spin and charge conservation to obtain the form of the fluctuation of the node distribution function $\delta \hat{f}_{N}$. This fluctuation leads to the total fluctuation in the current given by

$$
\Delta I_{1}(t)=\operatorname{Sp}\left[\hat{V}_{1} \delta \hat{I}_{1}(t)+\hat{V}_{2} \delta \hat{I}_{2}(t)\right]
$$

with

$$
\hat{V}_{n}=-\frac{(-1)^{n}}{2} \hat{1}+\frac{p}{4} \frac{\left(\mathbf{m}_{2}-\mathbf{m}_{1}\right) \eta_{r}+\left(\mathbf{m}_{2} \times \mathbf{m}_{1}\right) \eta_{i}}{|\eta|^{2} \cos ^{2} \frac{\theta}{2}+\eta_{r} \sin ^{2} \frac{\theta}{2}} \cdot \hat{\boldsymbol{\sigma}},
$$

where $\eta_{r}\left(\eta_{i}\right)$ is the real (imaginary) part of the relative mixing conductance $\eta=2 g^{\uparrow \downarrow} /\left(g^{\uparrow}+g^{\downarrow}\right)$. $\hat{V}_{n}$ is related to the average zero temperature distribution function $\hat{f}_{N}^{\mathrm{a}}$ by $\hat{V}_{n}=\left(\hat{f}_{1}-\hat{f}_{2}\right)\left(\hat{f}_{n}-\hat{f}_{N}^{\mathrm{a}}\right)$, where $\hat{f}_{n}$ is the distribution function in the $n$th ferromagnetic reservoir for energies between the chemical potentials of the reservoirs. (Because the reservoirs are held at thermal equilibrium at zero temperature, the allowed values for $\hat{f}_{n}$ are $\hat{1}$ and $\hat{0}$-the unit $2 \times 2$ matrix and the zero $2 \times 2$ matrix) $\mathrm{Sp}$ denotes the trace in spin indices to distinguish it from the trace in the space of transverse channels which we denoted Tr in the preceding discussion. Eq. (30) is valid in both the elastic and the inelastic regimes when the conductance matrix parameters are energy independent on the scale defined by the voltage bias.

Using Eq. (30) we express the time-dependent current fluctuations in terms of the Langevin sources $\delta \hat{I}_{i}(t)$. Then we apply Eq. (19) to find correlators for these Langevin sources and finally find the shot noise from Eq. (1):

$$
S=\frac{2 e^{2}}{h} \int_{\mu_{0}}^{\mu_{0}+|e V|} d E \operatorname{Sp}\left[\hat{S}_{1}(E)+\hat{S}_{2}(E)\right]
$$




$$
\begin{aligned}
\hat{S}_{n}(E)= & \sum_{\alpha \beta} \hat{g}^{\alpha \beta}\left\{\hat { f } _ { N } ( E ) [ \hat { 1 } - \hat { f } _ { N } ( E ) ] \left(2 \hat{V}_{n} u_{n}^{\beta} \hat{V}_{n} u_{n}^{\alpha}\right.\right. \\
& \left.\left.-u_{n}^{\beta} \hat{V}_{n} \hat{V}_{n} u_{n}^{\alpha}\right)-\left[\hat{f}_{N}(E)-\hat{f}_{n}\right]^{2} u_{n}^{\beta} \hat{V}_{n} \hat{V}_{n} u_{n}^{\alpha}\right\} \\
& +\sum_{\alpha \alpha^{\prime} \beta \beta^{\prime}} \hat{s}^{\alpha \beta \alpha^{\prime} \beta^{\prime}}\left\{u_{n}^{\alpha}\left[\hat{f}_{N}(E)-\hat{f}_{n}\right]\right. \\
& \left.\times u_{n}^{\alpha^{\prime}} \hat{V}_{n} u_{n}^{\beta}\left[\hat{f}_{N}(E)-\hat{f}_{n}\right] u_{n}^{\beta^{\prime}} \hat{V}_{n}\right\}
\end{aligned}
$$

In each sum $\alpha, \alpha^{\prime}, \beta$, and $\beta^{\prime}$ are spin indices, $u_{n}^{\alpha}=$ $\left(\hat{1}+\alpha \mathbf{m}_{n} \cdot \hat{\boldsymbol{\sigma}}\right) / 2$ are projection matrices corresponding to the magnetization direction $\mathbf{m}_{n}$ of the $n$th reservoir, $\hat{f}_{N}(E)$ is the distribution function on the normal metal island and $\hat{g}^{\alpha \beta}=\operatorname{Tr}\left[\hat{1}-r^{\alpha}\left(r^{\beta}\right)^{\dagger}\right]$ and $\hat{s}^{\alpha \beta \alpha^{\prime} \beta^{\prime}}=\operatorname{Tr}[\hat{1}-$ $\left.r^{\alpha}\left(r^{\alpha^{\prime}}\right)^{\dagger} r^{\beta}\left(r^{\beta^{\prime}}\right)^{\dagger}\right]$ are elements of the conductance matrix $\hat{g}$ (18) and shot noise $\hat{s}$ matrix (20).

All the terms in the sums of Eq. (31) are not independent: The elements of the first sum can be grouped to form a $2 \times 2$ Hermitian matrix while the elements of the second sum can be grouped to form a $4 \times 4$ Hermitian matrix with the same structure as the shot-noise $\hat{s}$ matrix (21).

In the following subsections we directly apply Eq. (31) to the cases of ballistic, diffusive, and tunnel junctions in both elastic and inelastic regimes.

\section{A. Elastic transport}

\section{Ballistic junctions}

The Fano factor for the collinear configurations of the ferromagnetic magnetizations can be found by applying the result for all-normal metal systems (9) for two independent spin channels

$$
\begin{aligned}
& F(\theta=0)=\frac{1}{4}, \\
& F(\theta=\pi)=\frac{1-p^{2}}{4} .
\end{aligned}
$$

The Fano factor is decreased in the antiparallel configuration $\theta=\pi$ when each spin component experiences an asymmetric double barrier system. The angular dependence of the Fano factor is shown in Fig. 3 for a set of different polarizations $p$.

For polarizations $p$ below the critical value $p_{c}=1 / 3$, the angular dependence of the Fano factor is monotonic. When $p$ exceeds $p_{c}$, there is a maximum in $F$ which continuously (and monotonically) increases from $\theta=0$ to $\theta=\pi$ as $p$ increases from $p_{c}$ to $1 . F_{\max }(p)$ monotonically increases from $1 / 4$ to 1 when $p$ is increased from $p_{c}$ to 1 . The position of the maximum thus gives another independent measurement of the polarization $p$ together with the ratio $F(\theta=\pi) / F(\theta=0)$.

It is interesting to note the limiting behavior of $F$ when $\theta$ approaches $\pi$ and $p$ approaches 1: $\lim _{p \rightarrow 1} \lim _{\theta \rightarrow \pi} F(\theta, p)=0$ and $\lim _{\theta \rightarrow \pi} \lim _{p \rightarrow 1} F(\theta, p)=$
1. Thus, for half-metallic ferromagnets $(p=1)$, there is a sharp drop in $F$ from 1 to 0 when $\theta$ approaches $\pi$.

In the exact antiparallel configuration, $\theta=\pi$, the Fano factor vanishes when the system is close to being half-metallic ( $p$ is close 1$)$ since the transport properties are governed by the barrier with the lower transmission which has a vanishingly small Fano factor for ballistic junctions. $p \approx 1$ means that one barrier has vanishing conductance and, therefore, the current and the shot noise vanish in the system. Relaxing the assumption of antiparallel magnetization when the relative angle is slightly below $\pi$, the Fano factor increases to 1 as some of the low transmission channels form. These low transmission channels determine the transport properties and the system effectively behaves as a single tunnel barrier system with $F \approx 1$.

\section{Diffusive junctions}

As discussed in the Introduction, the Fano factor has the universal value of $1 / 3$ in the collinear configuration for diffusive junctions. Thus we have from Eq. (8) for two spin-channels

$$
\begin{aligned}
& F(\theta=0)=\frac{1}{3}, \\
& F(\theta=\pi)=\frac{1}{3} .
\end{aligned}
$$

The $1 / 3$ suppression also holds for arbitrary angles when $p=0$ so that there is no spin accumulation. In general, there is an angular dependence of the Fano factor since the spins are coherent on the normal metal portion of the junctions. We show this dependence in Fig. Q 4 for $g_{N} / g=4$ and several values of $p$. When $p$ is finite, there is a minimum in $F(\theta)$. The position of the minimum is relatively insensitive to the value of the polarization $p$. When $g_{N} / g$ increases, this minimum shifts to larger angles and the Fano factor flattens in the limit of $g_{N} / g \rightarrow \infty$ (in this limit the junctions are fully ferromagnetic): $F(\theta)=1 / 3$ for $\theta<\pi$ with a singularity at $\theta=\pi$ when $F$ has a sharp minimum which deepens to zero for $p=1$. This mimics the behavior of the ballistic systems. The Fano factor identically equals $1 / 3$ in a special case of $p=1$ and $g_{N} / g=2$ when $g_{F}^{\uparrow} / g_{N} \rightarrow \infty$ and $g_{F}^{\downarrow} / g_{N} \rightarrow 0$, where $g_{F}^{\alpha}$ is spin- $\alpha$ conductance of the ferromagnetic portion of the junction [for a diffusive junction, in general, $\left.g^{\alpha}=g_{F}^{\alpha} g_{N} /\left(g_{F}^{\alpha}+g_{N}\right)\right]$.

\section{Tunnel junctions}

In this regime, the Fano factor displays a simple analytic dependence on the angle $\theta$ for any polarization $p$ :

$$
F(\theta)=\frac{1}{2}\left(1+p^{2} \sin ^{2} \frac{\theta}{2}\right) .
$$


The angular dependence is thus a simple monotonic interpolation between the values of the Fano factor in the collinear configurations [from Eq. (7) for two spinchannels]

$$
\begin{aligned}
& F(\theta=0)=\frac{1}{2} \\
& F(\theta=\pi)=\frac{1+p^{2}}{2}
\end{aligned}
$$

For completeness, we plot the corresponding Fano factor in Fig. 5.

\section{B. Inelastic transport}

\section{Ballistic junctions}

The collinear configuration in this regime gives a vanishing Fano factor

$$
\begin{aligned}
& F(\theta=0)=0 \\
& F(\theta=\pi)=0
\end{aligned}
$$

Also when the polarization $p=0$, the Fano factor $F$ vanishes for any angle $\theta$. But in the case of noncollinear configuration and nonzero polarization $p$, the Fano factor $F$ is always finite. This can be understood as follows: In general, the time-dependent distribution function of the normal metal node has a FD form for the two spin orientations along some (time-dependent) direction $\mathbf{u}(t)$. When there is a spin accumulation on the node, the chemical potentials corresponding to the electrons with spin parallel and antiparallel to $\mathbf{u}(t)$ are different. The electrons are injected into the normal metal island with spin orientations along the magnetization direction of the corresponding reservoir, $\mathbf{m}_{1}$ or $\mathbf{m}_{2}$. This means that these projected components of the distributions functions may be partially occupied. Partial occupation of the states effectively results in a 'thermal noise' contribution to the shot noise of the system. Consequently, the Fano factor is finite for intermediate angles $\theta$. This mimics the mechanism for nonzero shot noise in the all-normal metal elastic double barrier system with ballistic junctions which we discussed in the Introduction.

The analytic form of the Fano factor $F(\theta)$ is relatively simple:

$$
F(\theta)=\frac{p^{2} \sin \theta \cos \frac{\theta}{2}\left[1+|p|\left(1+2 \sin \frac{\theta}{2}\right)\right]}{8\left(1-|p| \sin ^{2} \frac{\theta}{2}\right)\left(1+|p| \cos ^{2} \frac{\theta}{2}\right)^{2}} .
$$

In Fig. 6 we show the angular dependence of the Fano factor for a set of different polarizations $p$. When $p$ increases from 0 to $1, F(\theta)$ yields a single maximum which monotonically shifts from $\theta=0$ to $\theta=\pi$. $F_{\max }(p)$ monotonically increases from 0 to 1 , when $\mathrm{p}$ is varied from 0 to 1 . The limiting behavior found in the elastic regime is also valid in the inelastic regime.

\section{Diffusive junctions}

In the collinear configuration the Fano factor is

$$
\begin{aligned}
& F(\theta=0)=\frac{1}{6} \\
& F(\theta=\pi)=\frac{1+p^{2}}{6} .
\end{aligned}
$$

The $1 / 6$ suppression also holds for any $\theta$, when $p=0$. We show the angular dependence of the Fano factor for a set of different polarizations $p$ and $g_{N} / g=4$ on Fig. 7 . For nonzero $p, F(\theta)$ has two local extrema, one minimum and one maximum, the minimum corresponding to a higher $\theta$. As in the elastic case, the angles corresponding to the extrema in $F(\theta)$ are relatively insensitive to the value of the polarization $p$. The function $F(\theta)$ becomes monotonic for sufficiently small $g_{N} / g$. In the opposite limit, when this ratio goes to infinity, $g_{N} / g \rightarrow \infty$, the junctions become completely ferromagnetic. In this limit, $F(\theta)=1 / 6$ for $\theta<\pi$ with a jump to $\left(1+p^{2}\right) / 6$ at $\theta=\pi$ much like in the elastic regime: Before the steep rise, the Fano factor $F$ has a sharp minimum at $\theta=\pi$ which deepens to zero for $p=1$.

\section{Tunnel junctions}

As was noted in the Introduction for the case of allnormal metal systems, the elastic and inelastic transport regimes yield the same Fano factor. We obtain that this remains valid for $F-N-F$ systems even in the case of noncollinear configuration, i.e. $F$ is given by Eq. (32).

\section{CONCLUSIONS AND DISCUSSIONS}

A semiclassical circuit theory for the shot noise in multiterminal ferromagnet-normal metal systems has been developed. The current is governed by 4 real parameters for each contact and the shot noise is governed by 11 real parameters. The shot-noise parameters have been evaluated for diffusive, tunnel, and ballistic junctions.

The circuit theory has been applied to symmetric double barrier $F-N-F$ systems. Both the elastic and inelastic regimes have been studied. In each of the regimes, a complex nonmonotonic angular dependence of the Fano factor was found in the case of diffusive junctions. In the case of ballistic junctions with large conductance polarization, a sharp drop of the Fano factor from 1 to 0 was found when the magnetization of the ferromagnets approaches the antiparallel configuration. Simple sinusoidal angular dependence of the Fano factor was found for the tunnel junctions in both the elastic and inelastic regimes.

We have in this work disregarded spin-flip processes. Spin-flip scattering can occur both in the junctions and on the nodes and these different processes will influence 
the current and the shot noise in different ways. The spin-flip processes on the nodes can be disregarded when the spin-flip relaxation time is longer than the transport dwell time $\tau_{\mathrm{sf}} \gg \tau_{d}$. For shorter spin-flip scattering times, the angular dependence of the average current is governed by a reduced effective polarization and a reduced mixing conductance. 6 We expect similarly that the shot noise is governed by a reduced effective polarization and a reduced effective mixing conductance. The anticipated effect of spin-flip scattering in the normal metal node on the Fano factor is thus mostly quantitative; the effective polarization and mixing conductance are reduced making the angular dependence weaker, and the angular dependence eventually vanishes when $\tau_{\text {sf }} \ll \tau_{d}$. (A discussion of the spin-flip scattering time $\tau_{\mathrm{sf}} v s$ the transport dwell time $\tau_{d}$ has been given by Brataas et al. in Ref. 7). Spin-flip scattering in the junctions can be disregarded when the junction is smaller in the transport direction than the spin-flip scattering length. If that is not the case, spin-flip scattering in the junction can have a qualitative effect as well as a quantitative effect both on the current and the shot noise. First, we also expect that the effective polarization and mixing conductance decrease with increasing spin-flip scattering in the contact. There is also a second, qualitative effect: In the absence of spin-flip scattering in the junctions, the cur- rent between a ferromagnetic node and a normal metal is determined by a $2 \times 2$ conductance matrix (18) and the fluctuations in the current are governed by a $4 \times 4$ shot-noise matrix (20). However, these matrices do not completely determine the current and shot noise in the presence of spin-flip scattering in the junction since more combinations of the scattering matrices become accessible (for the calculation of the average current see e.g. the appendix in Ref. (6). Consequently, an expanded basis of conductance and shot-noise parameters must be used and the angular dependence of both the current and the shot noise can be qualitatively different than in the case of no spin-flip scattering in the junctions.

\section{Acknowledgments}

We are grateful to G. E. W. Bauer, W. Belzig, B. I. Halperin, D. Huertas Hernando and Yu. V. Nazarov for stimulating discussions. This work was supported in part by the Norwegian Research Council, NSF Grant No. DMR 99-81283, the Schlumberger Foundation, and the NEDO International Joint Research Grant Program "Nano-magnetoelectronics",
1 R. Meservey and P. M. Tedrow, Phys. Rep. 238, 173 (1994).

2 P. M. Levy, Solid State Phys. 47, 367 (1994)

${ }^{3}$ M. A. M. Gijs and G. E. W. Bauer, Adv. Phys. 46, 285 (1997)

4 G. A. Prinz, Science 282, 1660 (1998).

${ }^{5}$ M. Johnson and R. H. Silsbee, Phys. Rev. Lett. 55, 1790 (1985); M. Johnson, ibid. 70, 2142 (1993); Science 260, 320 (1993).

6 A. Brataas, Yu. V. Nazarov, and G. E. W. Bauer, Phys. Rev. Lett. 84, 2481 (2000); Eur. Phys. J. B 22, 99 (2001).

7 J. Barnas and A. Fert, Europhys. Lett. 44, 85 (1998); A. N. Korotkov and V. I. Safarov, Phys. Rev. B 59, 89 (1999); A. Brataas, Yu. V. Nazarov, J. Inoue, and G. E. W. Bauer, ibid. 59, 93 (1999); Eur. Phys. J. B 9, 421 (1999); H. Imamura, S. Takahashi and S. Maekawa, Phys. Rev. B 59, 6017 (1999); A. Brataas and X. H. Wang, Phys. Rev. B 64, 104434 (2001).

8 Ya. M. Blanter and M. Büttiker, Phys. Rep. 336, 1 (2000).

9 B. R. Bulka, J. Martinek, G. Michalek, and J. Barnas, Phys. Rev. B 60, 12246 (1999); B. R. Bulka, ibid. 62, 1186 (2000).

10 E. R. Nowak, M. B. Weissman, and S. S. P. Parkin, Appl. Phys. Lett. 74, 600 (1999).

11 J. S. Moodera and L. R. Kinder, J. Appl. Phys. 79, 4724 (1996).

12 J. C. Slonczewski, Phys. Rev. B 39, 6995 (1989).
13 M. Büttiker, Phys. Rev. Lett. 57, 1761 (1986).

14 C. W. J. Beenakker and M. Büttiker, Phys. Rev. B 46, 1889 (1992); M. J. M. de Jong and C. W. J. Beenakker, Physica A 230, 219 (1996).

15 S. Oberholzer, E. V. Sukhorukov, C. Strunk, C. Schönenberger, T. Heinzel, and M. Holland, Phys. Rev. Lett. 86, 2114 (2001).

16 Yu. V. Nazarov, Phys. Rev. Lett. 73, 134 (1994).

17 D. H. Hernando, Yu. V. Nazarov, A. Brataas, and G. E. W. Bauer, Phys. Rev. B 62, 5700 (2000).

18 G. E. W. Bauer, Phys. Rev. Lett. 69, 1676 (1992); M. J. M. de Jong and C. W. J. Beenakker, ibid. 74, 1657 (1995).

19 P. W. Brouwer and C. W. J. Beenakker, J. Math. Phys. 36, 4904 (1996).

20 X. Waintal, E. B. Myers, P. W. Brouwer, and D. C. Ralph, Phys. Rev. B 62, 12317 (2000).

21 A. D. Stone, P. A. Mello, K. A. Muttalib, and J.-L. Pichardin Mesoscopic Phenomena in Solids, edited by B. L. Altshuler, P. A. Lee, and R. A. Webb (North Holland, Amsterdam, 1991).

22 M. J. M. de Jong and C. W. J. Beenakker, Phys. Rev. B 51, 16867 (1995); E. V. Sukhorukov and D. Loss, ibid. 59, 13054 (1999).

${ }^{23}$ K. Xia, P. J. Kelly, G. E. W. Bauer, A. Brataas, and I. Turek, cond-mat/0107589 (unpublished). 


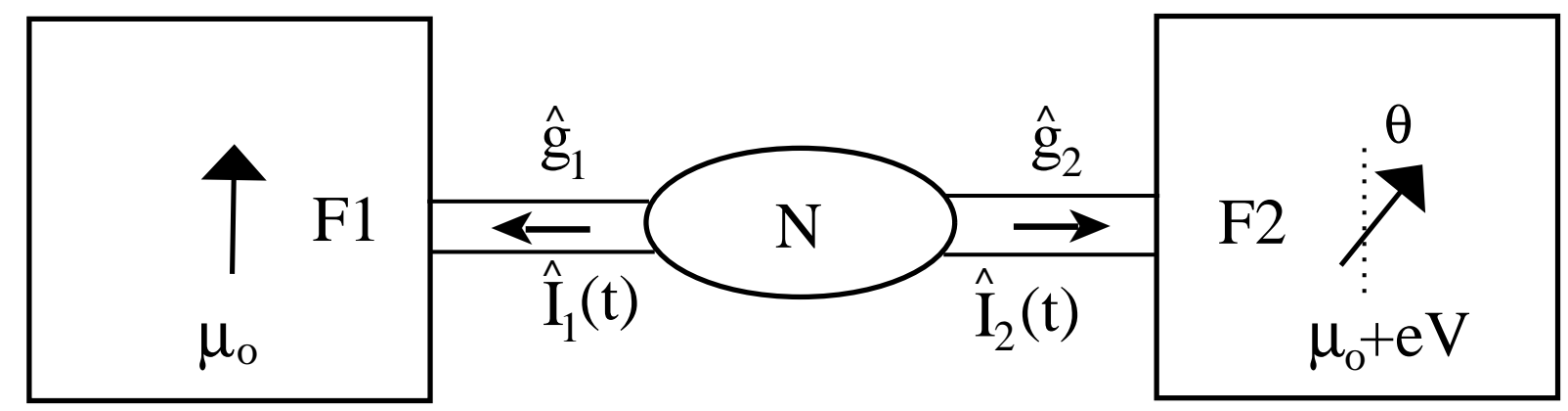

FIG. 1: The two-terminal device consisting of a normal metal node $(N)$ attached to two ferromagnetic reservoirs $(F 1$ and $F 2)$ with arbitrary relative magnetization direction $\theta$. A source-drain bias $V$ is applied between the ferromagnetic reservoirs and time-dependent currents $\hat{I}_{1}(t)$ and $\hat{I}_{2}(t)$ flow into the normal metal island from the reservoirs $F 1$ and $F 2$, respectively. The contact between the ferromagnetic $F 1(F 2)$ and the normal metal node is characterized by the conductance matrices $\hat{g}_{1}\left(\hat{g}_{2}\right)$.

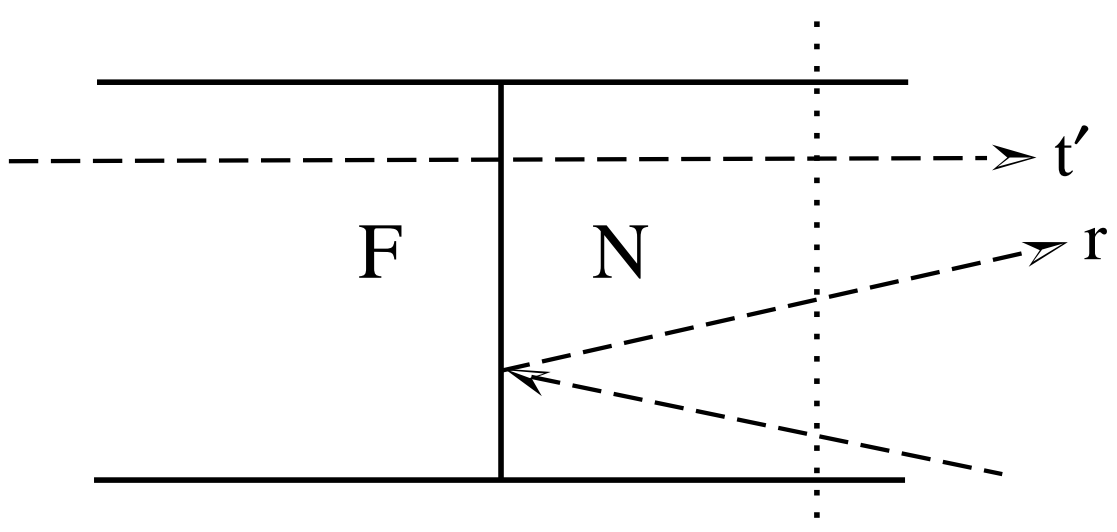

FIG. 2: A contact between a ferromagnetic node and a normal metal node. The current is evaluated at the normal metal side (dotted line). The transmission coefficient from the ferromagnet to the normal metal is $t^{\prime}$ and the reflection matrix from the normal metal to the normal metal is $r$. 


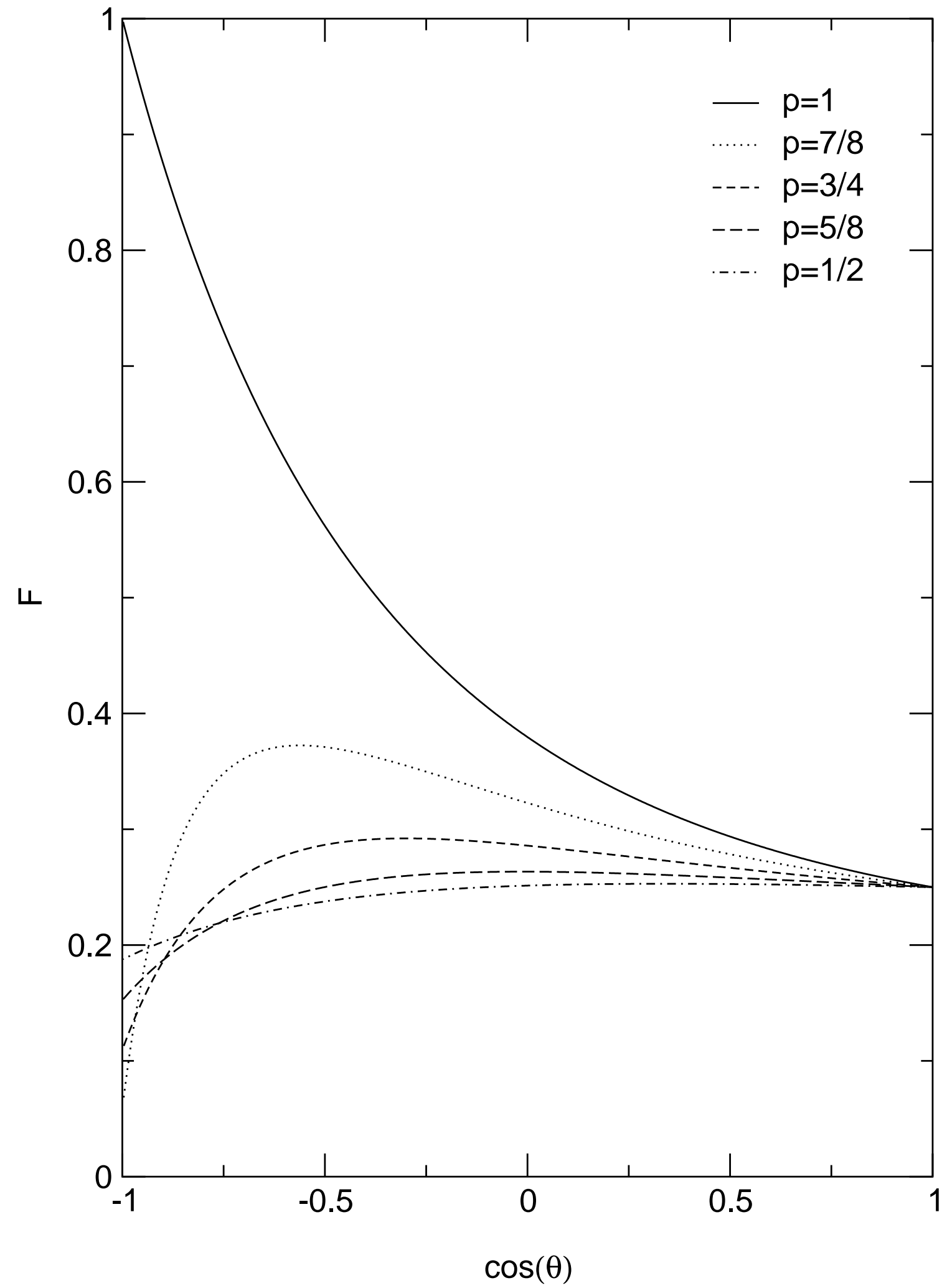

FIG. 3: Angular dependence of the Fano factor $F$ in $F-N-F$ systems in the elastic regime with ballistic junctions. The results are shown for different polarizations $p=\left(g^{\uparrow}-g^{\downarrow}\right) /\left(g^{\uparrow}+g^{\downarrow}\right)$. 


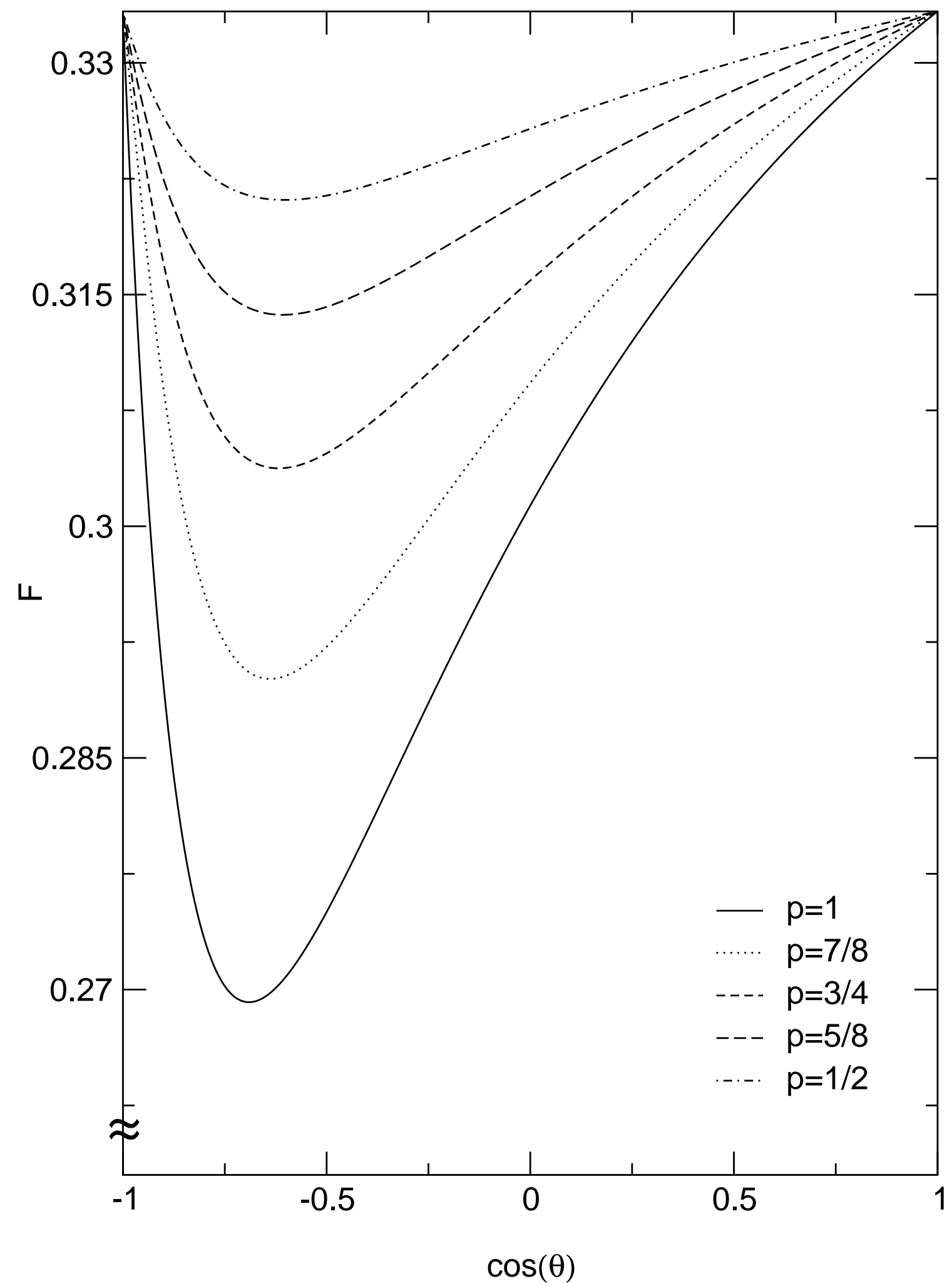

FIG. 4: Angular dependence of the Fano factor $F$ in $F-N-F$ systems in the elastic regime with diffusive junctions. The results are shown for different polarizations $p=\left(g^{\uparrow}-g^{\downarrow}\right) /\left(g^{\uparrow}+g^{\downarrow}\right) \cdot g_{N} / g=4$. 


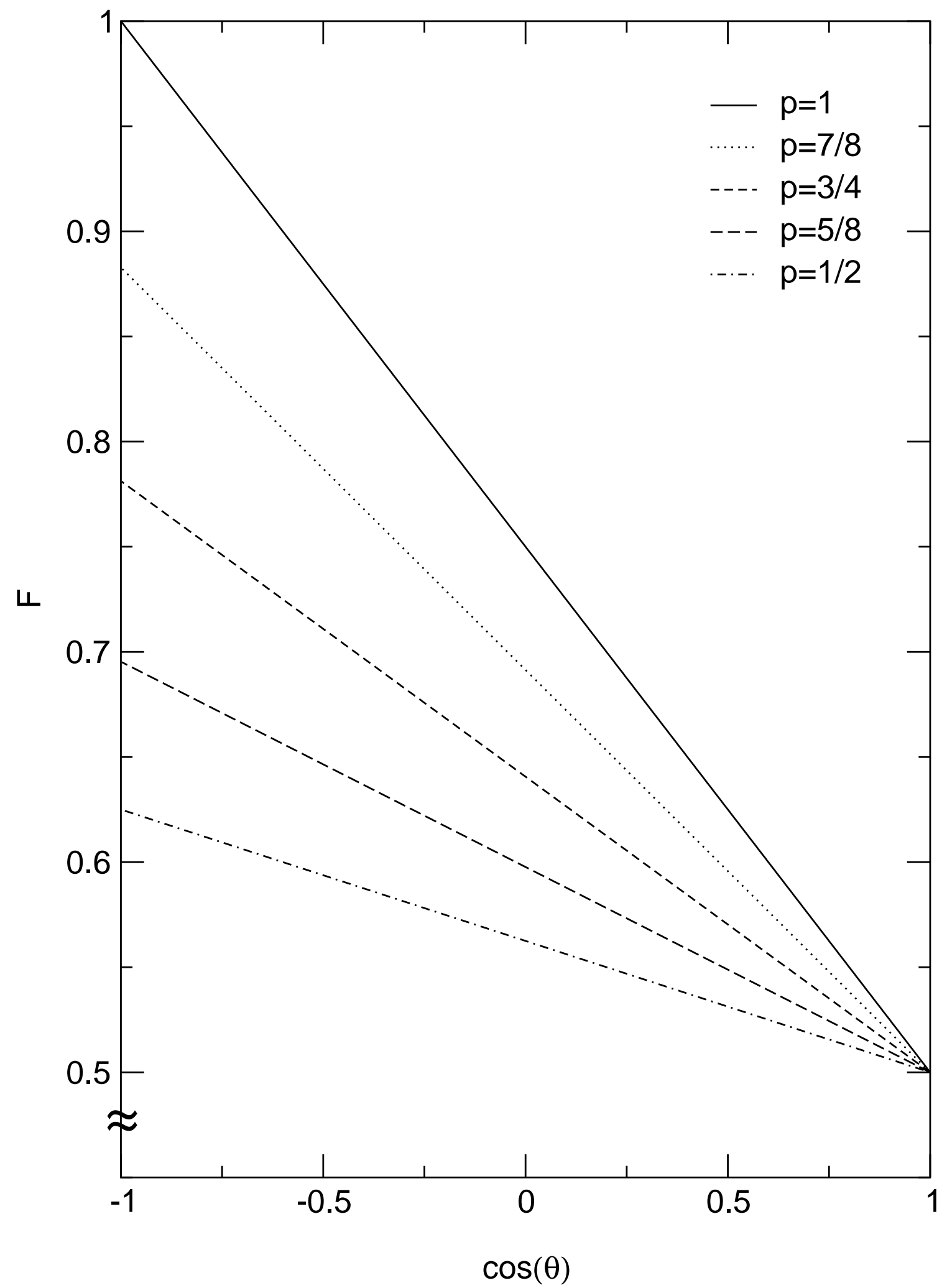

FIG. 5: Angular dependence of the Fano factor $F$ in $F-N-F$ systems with tunnel junctions. The results are shown for different polarizations $p=\left(g^{\uparrow}-g^{\downarrow}\right) /\left(g^{\uparrow}+g^{\downarrow}\right)$. 


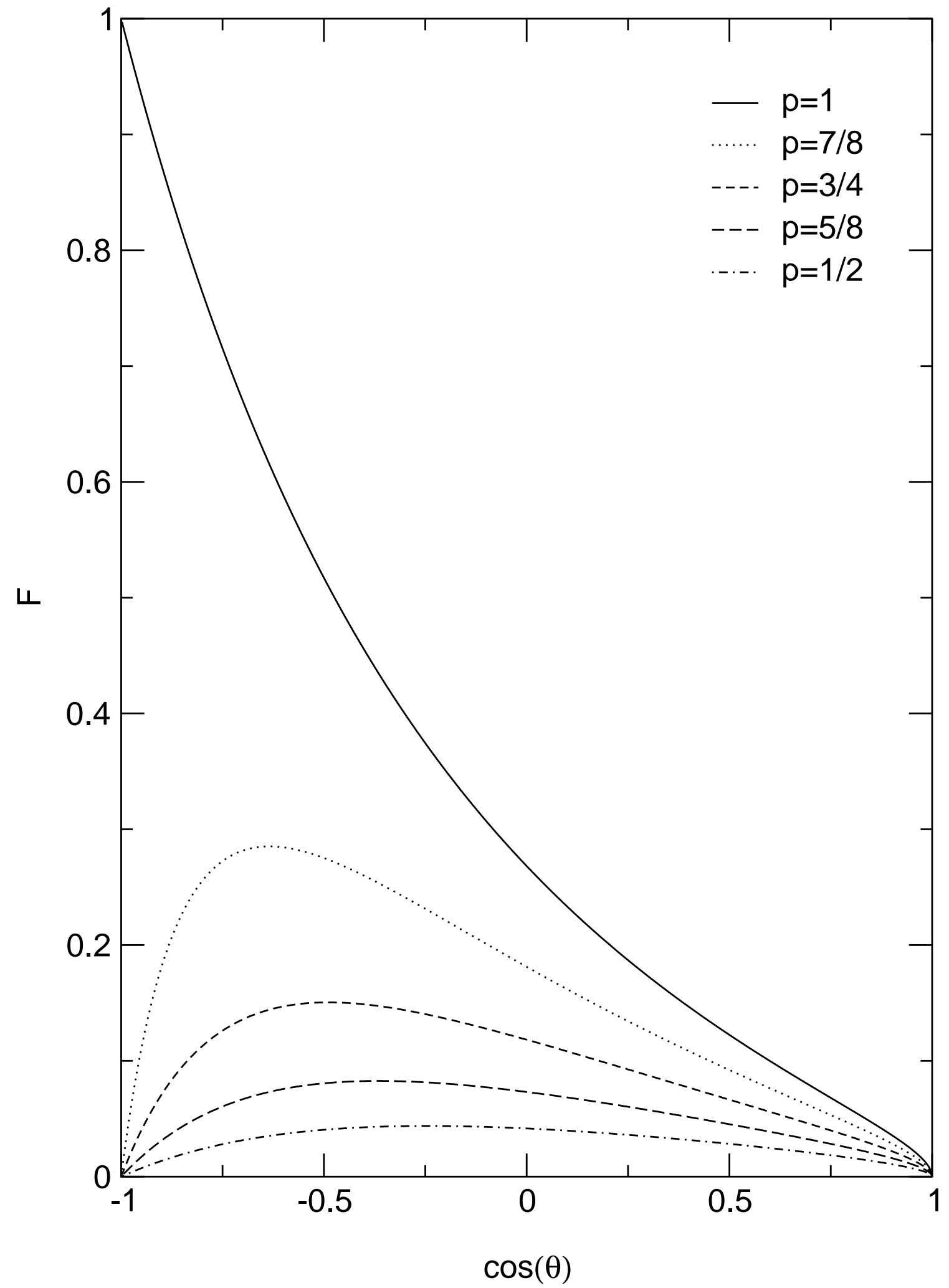

FIG. 6: Angular dependence of the Fano factor $F$ in $F-N-F$ systems in the inelastic regime with ballistic junctions. The results are shown for different polarizations $p=\left(g^{\uparrow}-g^{\downarrow}\right) /\left(g^{\uparrow}+g^{\downarrow}\right)$. 


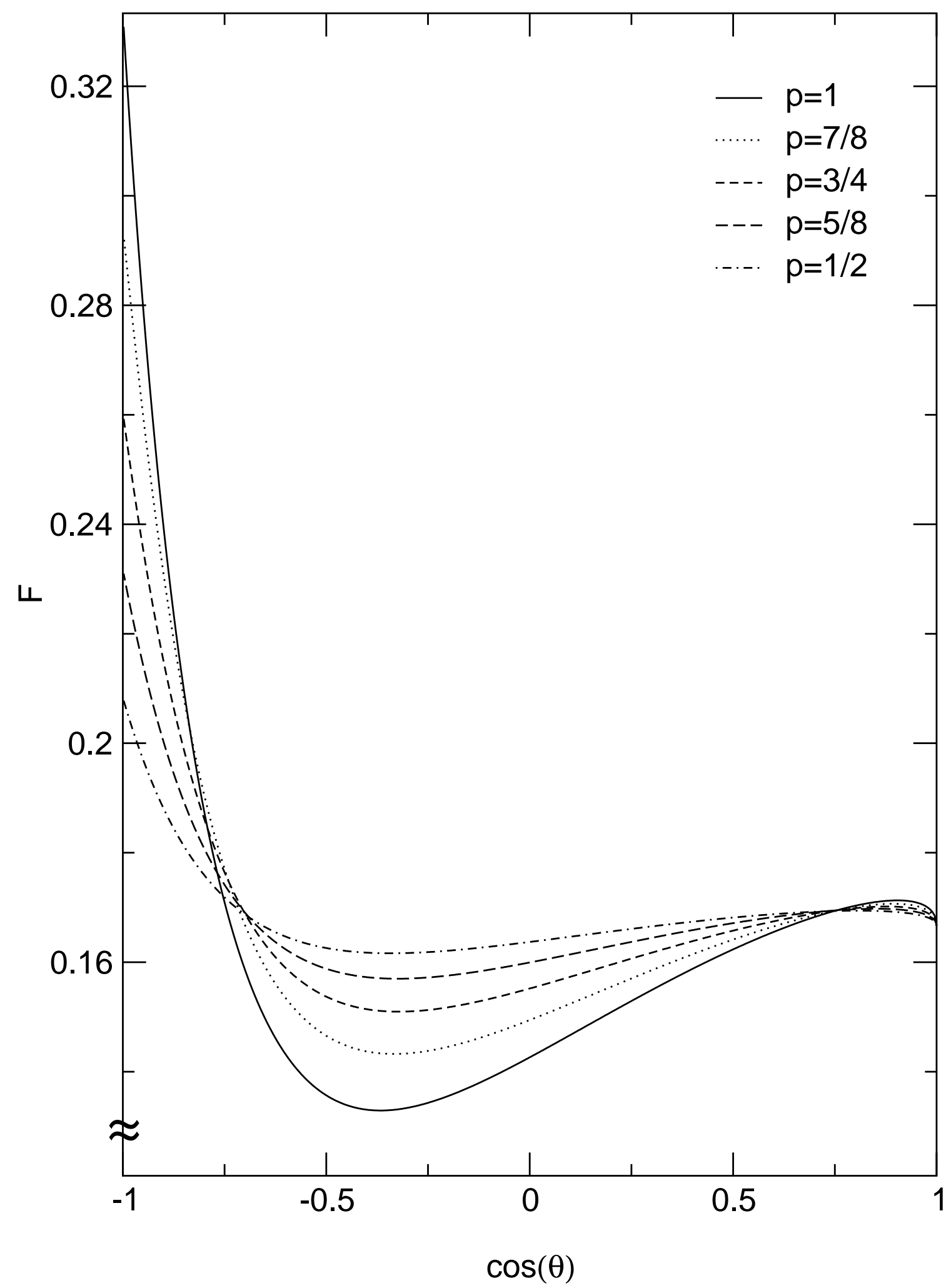

FIG. 7: Angular dependence of the Fano factor $F$ in $F-N-F$ systems in the inelastic regime with diffusive junctions. The results are shown for different polarizations $p=\left(g^{\uparrow}-g^{\downarrow}\right) /\left(g^{\uparrow}+g^{\downarrow}\right) \cdot g_{N} / g=4$. 